\title{
Study of Indoor LTE Green Small-Cells Using Mobile Fronthaul Architecture over Hybrid Fiber-Wireless Channels
}

\author{
Y. Hazan, M. Ran and E. Omiyi \\ Department of Electrical and Electronics Engineering, \\ Holon Institute of Technology, Holon, Israel \\ E-mail: yaronhazan@gmail.com; \{mran; eitano\}@hit.ac.il
}

Received 31 August 2016; Accepted 16 November 2016;

Publication 19 December 2016

\begin{abstract}
6 Gbps transmission over a very low-power multi Radio Access air-interface for indoor is demonstrated. A single-mode fiber based fronthaul architecture is employed together with plastic optical fiber in indoor small-cells. The low power transmission coverage is similar to the classic femtocell coverage and is suitable for most $5 \mathrm{G}$ indoor scenarios.
\end{abstract}

Keywords: 4G, 5G, C-RAN, Hybrid Fiber-Wireless (HFW), Fronthaul, Green Radio (GR), LTE, Multi-Input Multi-Output (MIMO), Plastic Optical Fiber (POF), Radio Over Fiber (ROF), Visible Light Communication (VLC).

\section{Introduction}

The demand for efficient and inexpensive broadband mobile communication has increased steadily over the past decade and in an ever increasing rate. The telecom industry's current 5G standardization process defines several use cases for the $5 \mathrm{G}$ network. The data rate requirements for the indoor use case are $1.5 \mathrm{Gbps}$ in the Downlink (DL) channel and $500 \mathrm{Mbps}$ in the Uplink (UL) channel for a single user in the entire cell coverage, including at the cell edge [1]. The telecom operators are trying to meet this challenge

Journal of Green Engineering, Vol. 6_3, 257-284.

doi: 10.13052/jge1904-4720.632

(C) 2016 River Publishers. All rights reserved. 
with the fast adoption of new technologies [29]. Recently, the operators around the world have started to address the increasing demand for high bandwidth by adopting the $4 \mathrm{G}$ mobile technology known as LTE-A. The LTE-A standard (3 GPP Rel. 10 and beyond) adopted several tools to address the increasing demand for high bandwidth. These include the increase of the effective available channel bandwidth per user up to $100 \mathrm{MHz}$, usage of multiple antenna techniques (MIMO), and usage of diverse modulation and coding rate schemes. Furthermore, a new "small-cell" feature was added to the standard, which allows operators to dramatically increase the maximum throughput for the end user, as compared to what is achievable in a network without small-cells.

In recent years a new global trend, "Green Radio" (GR) [2], has emerged; increasing awareness of the need for power saving and environmentallyfriendly communication strategies. This environmental context is expressed by the demand for energy conservation and reduced exposure to electromagnetic radiation of any kind. This includes the reduction of radiation originating from the cellular networks and mobile communication devices. Several industryacademia research projects have been dedicated to GR, among them the 5-year GreenTouch [3] project under the European Framework Programme FP7 that ended in 2015. Furthermore, the 5G target is 2000 fold increase in the energy efficiency of the network over the next 10 years [1].

$5 \mathrm{G}$ networks are expected to offer efficient and cost-effective new services, such as tailored network solutions for vertical markets [1]. In contrast to the evolution of previous generations of mobile networks, the requirement of $5 \mathrm{G}$ for a flexible and diverse network architecture will require also a sophisticated integration of massive computing and storage infrastructures. For this purpose, the $5 \mathrm{G}$ architecture is designed to address this challenge by adopting new networking technologies and concepts such as Software Defined Networking (SDN) and Network Function Virtualization (NFV) [4]. These concepts include function splits in the Radio Access Network (RAN) and across the core network.

In addition, to better cope with the Internet usage shift from host-centric end-to-end communication to receiver-driven content retrieval, innovative green information-centric networking (ICN) architectures have been proposed [30]. Formally, the ICN approach is designed to evolve the Internet infrastructure to support a communication model of accessing data by name, regardless of the origin server location. This approach is used by introducing uniquely named data as a core principle. Therefore, data becomes independent from location, application, storage, and means of transportation, enabling 


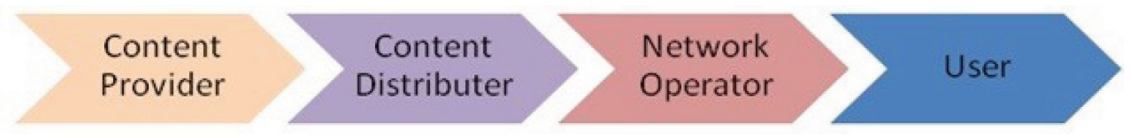

Figure 1 ICN reference architecture model [36].

in-network caching and replication. The expected benefits are improved efficiency, better scalability with respect to information/bandwidth demand and better robustness. Although the benefits of the ICN model are yet to be proven as the benefits from the main feature of caching were challenged [8], the ICN model is still attractive as an additional alternative model in the context of sharing the network resources. Therefore, in the context of this paper the ICN reference model can be envisioned either as an architecture where the data stored at the content providers becomes independent from location by accessing the data by name and as a consequence enabling in-network caching and replication or as a more basic architecture as suggested in Figure 1 which illustrates the ICN reference architecture model, where the data from the content distributors can be aggregated to a central focal point that will be shared by all network operators. This in turn, will reduce the redundant infrastructure between each of the network operators to the content distributers. Eventually the ICN reference model of choice can be decided based on the design that achieve the most benefits in the best possible way, the ICN reference model of choice is merely an optimized variant of the ICN reference architecture model.

In this paper, we will explore a possible solution that meets $5 \mathrm{G}$ requirements for indoor scenarios. The main ideas of this paper are as follows:

1. Indoor small-cells to achieve the beyond-4G and $5 \mathrm{G}$ capacity and density requirements.

2. Fronthaul architecture, which includes the baseband unit (BBU) at the logistic center (i.e., Radio Cloud Center, RCC [5] or Cloud-RAN [6]) and the remote radio head (RRH) installed at home/office, to comply with the required coverage while keeping the operators OPEX minimal.

3. Connecting the BBU and the RRH with optical fibers: single-mode fiber between the logistic center to the customer building and with Plastic Optical Fiber (POF) inside the building. Using the digital radio-overfiber (D-RoF) technique for leveraging the decentralization of the RRH concept without any degradation in the potential of the quality of service level from the small-cell and with minimal cost. 
4. Taking advantage of the fronthaul architecture for indoor coverage improvement allows to effectively reduce the transmission power dramatically ( $\sim 40 \mathrm{~dB})$, while maintaining the quality and reliability levels of the communication.

5. Flexible and dynamic RRH architecture to support $5 \mathrm{G}$ concepts of SDN and NFV.

6. Multi-Radio Access Technology (RAT) based RRH for low-power, high speed, flexible and reliable Radio Access Network for meeting the 5G indoor requirements.

\section{Main Building Blocks}

\subsection{Fronthaul and C-RAN}

As the data capacity in networks continues to rise exponentially, mobile operators are looking for new architectures to reduce cost, simplify networks and share resources matched to the dynamic nature of the mobile networks. Power and space are a rare resources at the cell sites and the power bills for the operators are considerable.

Therefore, the operators are turning to every possibility to reduce these costs and space requirements. A new trend of mobile operators is to address these requirements by moving to the Centralized-RAN and eventually move to the Cloud-RAN (C-RAN) [5, 6, 31-35] architectures. Energy savings of $70 \%$ in the OPEX of the BS infrastructure for $2 \mathrm{G}$ and $3 \mathrm{G}$ using C-RAN was reported [7].

This is in sharp contrast to the classical architecture, where each cell is based on a standalone base station with both the digital and radio units installed at the cell site. The cell site is located inside a building with stable power supply and air conditioning. This architecture incurred high installation and operation costs, as well as long installation times. To efficiently manage the evolving complexity of the standards, a new distributed base station architecture was introduced. This architecture provides deployment flexibility, cost reduction, high capacity coverage and improved network performance.

In contrast to the classical architecture, the centralized RAN architecture physically splits the base station into two pools of components: Baseband Units (BBUs) and Remote Radio Heads (RRHs). The RRHs perform the analogue processing, and can be mounted next to the antenna. The BBUs perform the baseband digital processing, and are connected to the corresponding RRHs, usually by means of fiber technology. This new connectivity interface is called "Fronthaul". 
This modular design optimizes the space utilization and deployment times, and reduces some of the operator costs. The transition to fiber based connectivity between the BBUs and RRHs allows the possibility to dramatically increase the distance between the BBUs and the RRHs. Furthermore, it enables the relocation of the BBUs from the cell site into the network. Thus, centralizing stacked BBUs at the location of the logistic center.

This has some advantages on top of the operating and installation costs. One of the main benefits of the co-location of BBUs, is easy cooperative processing between adjacent cells. This is mainly as a consequence of the reduced delay between cells, which leads to better interference mitigation between cells, advanced MIMO techniques between base stations and more. Furthermore, the simplified interface between the BBUs and the core network enable increased security over the fronthaul link.

Ultimately, the last step in this architecture evolution is to integrate the stacked BBUs at the logistic center into a single larger BBU pool with load balancing. This architecture is the Cloud-RAN architecture, which allows load balancing across a number of RRHs and enables optimal resource allocation. The optimal resource allocation of the C-RAN concept manifests itself not only by sharing the resources of a single operator or a single network but also by sharing the resources between different operators or networks. The sharing between operators can be implemented by sharing only the pool of BBU's between the operators or even by sharing the same infrastructure starting from the BBU's, continuing with the fronthaul and ending in the RRH's or any other sharing combination.

This resource sharing can be software configurable, which enables ondemand allocation of infrastructure resources, and allows for maximum utilization of the infrastructure hardware. This in turn paves the way for a new business model, where ownership of the physical infrastructure will not lie with the traditional operators, but rather with a new infrastructure service provider. This service provider could be Greenfield cooperation or a joint venture of the operators. The infrastructure service provider can more efficiently allocate to the operators the exact resources that they need, by globally maximizing the utilization of the infrastructure. This would result in a reduction in energy consumption and total cost of ownership.

Furthermore, using the same infrastructure could pave the way for an efficient Information Centric-Networking (ICN) architecture by connecting the content distributors to the core shared network of the network operators. In this way, the data from the content distributors can be aggregated to a central 
focal point that will be shared by all network operators. This will reduce the redundant infrastructure between each of the network operators to the content distributers.

Regardless of the business model, the resource sharing can potentially increase power savings and economic benefits for the operators.

\subsection{POF}

Optical fibers, often high capacity single-mode fiber, are connected to the building up to the point of the home Residential Gateway (RG). Today, inbuilding broadband access is supported mainly by telephone lines or UTP cables, which has been shown [5] to be inadequate for the support of future broadband in-building networks. These future networks will be based on an optical infrastructure, and will support bandwidth consuming services, such as Ethernet services, as well as digital TV. The Plastic Optical Fiber (POF) has shown its significant advantages in the physical layer for the in-building environment, mainly because of its large diameter and material properties [9].

Because of the very large core diameter of the POF, the installation of POF links in the home can be very simple compared to a single-mode fiber. This enables simple installation and maintenance without relying on special training, significantly reducing the cost of the home network infrastructure.

POF systems also require low-cost optical transceivers, permitting costeffective solutions. Additional advantages of the POF network in terms of installation cost over other infrastructures have also been reported [10]. There are mainly two types of POF available in the market: poly-methylmethacrylate (PMMA) and perfluorinated (PF). The former is a high attenuation and low bandwidth first generation POF, while the latter is a lower attenuation and higher bandwidth next generation POF with typical core diameters ranging from $0.05 \mathrm{~mm}$ to $1 \mathrm{~mm}$.

Comparing these diameters with the $0.009 \mathrm{~mm}$ core diameter of singlemode fiber, the larger core diameter and the larger numerical aperture of the POF allow larger connector tolerances where small misalignments can be tolerated. In contrast, the alignment of the single-mode fiber must be very precise. The attenuation of the POF is higher compared to the single-mode fiber, while the bandwidth of the POF is much lower compared to the singlemode fiber. 


\subsection{Error-Correcting Codes for Fiber-Optic Communication Systems}

It is well known from the literature that standard error-ccorrecting codes for Fiber-Optic Communication Systems can dramatically improve the BER performance from $10^{-3}$ at the input to $10^{-12}$ at the output [11]. The first generation FEC, such as the ITU-T recommendation G.709, were based on the $\mathrm{RS}(255,239)$ Reed-Solomon code. This code achieves a coding gain of approximately $6.5 \mathrm{~dB}$. The second generation codes, such as the one introduced in Appendix I.3 of ITU-T recommendation G975.1, is based on a concatenated coding scheme with an outer $(3860,3824)$ binary BCH code and an inner $(2040,1930)$ binary BCH code. This code achieves a coding gain of approximately $9 \mathrm{~dB}$ [12]. While the first generation FEC, introduces $7 \%$ redundancy, compared to a second generation FEC, with $23 \%$ redundancy [13], higher latencies are needed to support the second generation FEC with two decoding iterations. Third generation codes, based on nonbinary LDPC codes, can provide redundancy of $12.6 \%$, providing a coding gain of more than $10.5 \mathrm{~dB}$ with a BER of $10^{-12}$ at the output [14].

\section{Suggested Architecture}

In practice, the $\mathrm{BBU}$ in a $\mathrm{C}-\mathrm{RAN}$ network can be connected to more than one RRH. In order for the data to be efficiently delivered to its destination the BBU and RRH should use a communication protocol. Currently the most promising available protocol for the BBU-RRH interface is based on the CPRI [15] (Common Public Radio Interface). The CPRI was last updated in 2015 and is expected to be updated in the future. The CPRI was originally designed as an interface to support the BBU-RRH connectivity, while transmitting the digital IQ data between any BBU to any RRH while supporting different BBU-RRH configurations. Furthermore, the current CPRI standard requires that the maximum BER will be better than $10^{-12}$ with line rates of more than 10137.6 Mbps per CPRI channel. The suggested architecture is based on the assumption that the BBU-RRH interface could deliver line rates of about 100 Gbps which represent LTE-A small-cell at full capacity. It is anticipated that in the future the CPRI standard will be updated to support such rates with the same overhead levels. Regardless of this assumption the suggested architecture could support current CPRI standard at $100 \mathrm{Gbps}$ rates assuming implementation of OTDM (Optical Time-Division Multiplexing) [16] over 
the fiber-optic channel while multiplexing up to 10 channels of standard 10137.6 Mbps CPRI channels. The actual net rates of the data over the CPRI can be calculated based on the following:

$$
R=M \times S C \times F F T \times N \times 2
$$

Where $\mathrm{M}$ represents the number of antennas in use (maximum of 8 ), $\mathrm{N}$ is the sampling width $(\mathrm{N}=15$ can be used for LTE), factor 2 results from the information in the In-phase and Quadrature, SC is the spacing between subcarriers $(15 \mathrm{KHz})$ and FFT is the FFT size (2048 for a maximum channel of $20 \mathrm{MHz}$ ). The CPRI rate in the digital section can be calculated using the following formula:

$$
R_{C P R I}=R \times C \times 16 / 15
$$

where $16 / 15$ refers to the protocol relative overhead and $C$ is the code rate. The following Table 1 demonstrates the net data rate and actual CPRI rates over the digital section for several LTE-A scenarios.

The calculations show that for Frequency-Division Duplexing (FDD) LTE-A air interface, the required CPRI rate is $40.55 \mathrm{Gbps}$ in each direction, with a total cell capacity of 99.8 Gbps and $23 \%$ FEC redundancy.

In the C-RAN architecture, multiple RRHs can be connected to the logistic center where the BBUs are located, with the resource sharing paradigm forbidding dedicating a specific BBU to a specific RRH. Therefore, the maximum allowed distance between the BBU and RRH is bounded. This distance is limited by the timing requirements of the LTE-A HARQ protocol, which is used as the retransmission mechanism between the base stations and the end users. The timing requirement is such that the end user needs to receive the ACK/NACK feedback within three LTE sub-frames, which is a duration of about 3 msecs. Therefore, the base station should complete the relevant processing within this $3 \mathrm{msec}$ time frame. Since the suggested architecture includes the CPRI in the model, the processing time and other

\begin{tabular}{|c|c|c|c|c|c|c|c|}
\hline \multicolumn{2}{|c|}{$\begin{array}{c}\text { LTE-A Channel } \\
\text { BW [MHz] }\end{array}$} & \multicolumn{2}{|c|}{$\begin{array}{c}\text { MIMO } \\
\text { Configuration }\end{array}$} & \multicolumn{2}{|c|}{$\begin{array}{c}\text { Desired } \\
\text { I/Q Rate [Gbps] }\end{array}$} & \multicolumn{2}{|c|}{$\begin{array}{c}\text { Desired } \\
\text { CPRI Rate [Gbps] }\end{array}$} \\
\hline DL & UL & DL & UL & DL & UL & DL & UL \\
\hline $5 * 20$ & $5 * 20$ & $8 \times 8$ & $8 \times 8$ & 36.864 & 36.864 & 40.55 & 40.55 \\
\hline $5 * 20$ & $5 * 20$ & $4 \times 4$ & $2 \times 2$ & 18.432 & 9.216 & 20.275 & 10.138 \\
\hline 20 & 20 & $2 \times 2$ & $2 \times 2$ & 1.843 & 1.843 & 2.4576 & 2.4576 \\
\hline
\end{tabular}
delays inherent in the fiber channel must be bounded by this limitation. 
This timing restriction can typically result in a processing time limitation of about $2.75 \mathrm{msec}$ for the base station and about $0.25 \mathrm{msec}$ for the fronthaul section.

Based on this budget, the maximum distance between the BBU and the RRH can be calculated. Assuming a round-trip transmission latency of $10 \mu \mathrm{s} / \mathrm{km}$, the maximum separation between the BBU and RRH is limited to $25 \mathrm{Km}$. This distance includes the distance of the entire fiber section i.e., the single-mode fiber and the POF. For the model to be efficient, both fibers will use the same wavelengths at the standard $C$ band i.e., $1550 \mathrm{~nm}$, resulting in a seamless transition between both fiber types. This results in lower costs, because of their compatibility with standard glass fiber transceivers and the absence of wavelengths conversions.

Figure 2 illustrates the suggested architecture with several RRHs and a BBU pool at the operator's central office. Apart from the rate consideration, there is another aspect to consider in the architecture design. This is the flexibility of the architecture. In terms of architecture flexibility, the suggested architecture is relatively flexible. The flexibility is inherent in the C-RAN

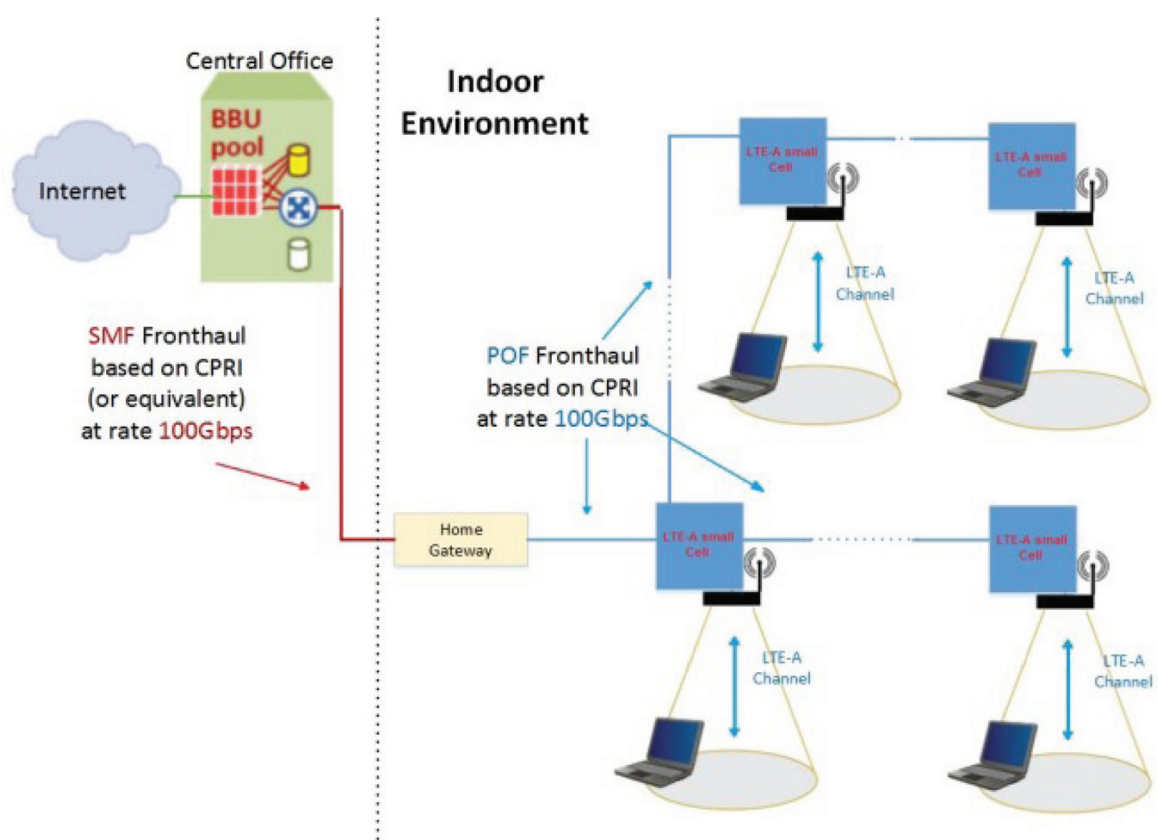

Figure 2 Suggested architecture. 
concept, mainly by means of using the BBU pool. In this way the infrastructure resources can be easily shared and dynamically re-allocated from idle to busy links. Moreover, the CPRI protocol supports several different topologies. This enables flexibility in RRH deployment with little or no changes to the POF installation. This flexibility can be software configurable and therefore supports the SDN and NFV 5G architecture concepts.

Another aspect to consider in the architecture design is the preferred location of the RRHs. Factors that determine the location of RRHs include the desired coverage, transmitted power, cross interference between the RRHs and the total cost. These factors are all influenced by the number of installed RRHs in a given area.

These factors are inherently contradictory. On one hand, increasing the transmitted power improves the coverage, and reduces the number of RRHs to be deployed, which in turn reduce cost. However, this may result in increased cross-interferences between the RRHs. Conversely, the increase in the number of RRHs to be deployed allows for decreasing the transmit power, and may reduce the potential for cross-interferences between the RRHs, but increases cost. Therefore, suboptimal compromise deployment solution is necessary, which aims at maximizing coverage, while imposing a limit on acceptable deployment cost per coverage area.

\section{Simulation Results and Discussion}

The simulation analysis has divided the system into two subsystems, in order to minimize complexity and simulation time. The first subsystem models the fiber-optic part of the network and the second models the wireless part. The main focus in the fiber-optic section is on the POF channel. This is because of the stringent requirement to achieve data rates of up to $100 \mathrm{Gbps}$, at a minimum BER of $10^{-12}$ and a total distance of $100 \mathrm{~m}$. The main focus in the wireless section is on indoor coverage and capacity, while using much lower transmit power than a traditional small-cell.

The BER requirements for the CPRI sets the target BER of the fiber channel to a level several orders of magnitude lower than the expected BER of the wireless channel. Therefore, the fiber channel is considered error free relative to the wireless channel. Therefore, the performance obtained from analyzing each channel separately is expected to be an accurate approximation of the performance obtained from the analysis of the real combined fiber/wireless channel. 


\subsection{The Optical Channel}

Real measurements of $112 \mathrm{Gbps}$ transmission over POF for $100 \mathrm{~m}$ were demonstrated [17]. In this work, a simulation based on the setup in [17], which is adjusted to the requirements in the model presented in this paper, was performed. The simulation validates that the required rates of $100 \mathrm{Gbps}$ over $100 \mathrm{~m}$, at BER exceeding $10^{-3}$ before FEC, can be achieved. This result establishes that the fiber channel is an error free channel compared to the wireless channel, within the bounds of the requirements for the proposed system architecture.

\subsection{The Wireless Channel}

The wireless part of the simulation compares the performance of the traditional small-cell deployment (used as a reference) to the small-cell deployment using the proposed architecture. The proposed architecture takes advantage of the C-RAN concept, with a centralized baseband unit at the operator's office coupled to remote radio heads that are deployed wherever desired. Each RRH can function as a full cell or as a partial cell, depending on demand. The wireless channel characteristics were simulated based on the A1 indoor scenarios of the WINNER (Wireless World Initiative New Radio) II model [18].

For both scenarios, a $20 \mathrm{MHz}$ LTE-A base station with a $2 \times 2 \mathrm{MIMO}$ configuration is assumed. This basic configuration is used in order to ease simulation complexity. The performance of more complex LTE-A configurations can be extrapolated with relative ease from this basic configuration. For example, consider a $100 \mathrm{MHz}$ LTE-A base station with a $2 \times 2 \mathrm{MIMO}$ configuration. In this configuration, 5 non-overlapping $20 \mathrm{MHz}$ channels are used. Since the channels are non-overlapping and located in the same frequency band, it is easy to show that the difference in capacity performance between each of the $20 \mathrm{MHz}$ channels is marginal. Therefore, for simplicity, it is reasonable to simulate the capacity for one channel and multiply it by 5 , in order to estimate the capacity of the $100 \mathrm{MHz}$ configuration.

Figure 3 illustrates the location of the traditional small-cell installation in an indoor scenario. In the traditional small-cell scenario, the transmit power is set to the maximum allowed by the LTE standard of $+20 \mathrm{dBm}$. The maximum power is used, in order to achieve the maximum capacity in every possible user location in the indoor environment. The capacity was considered as the maximum throughput achieved for a maximum BER of $10^{-6}$. 


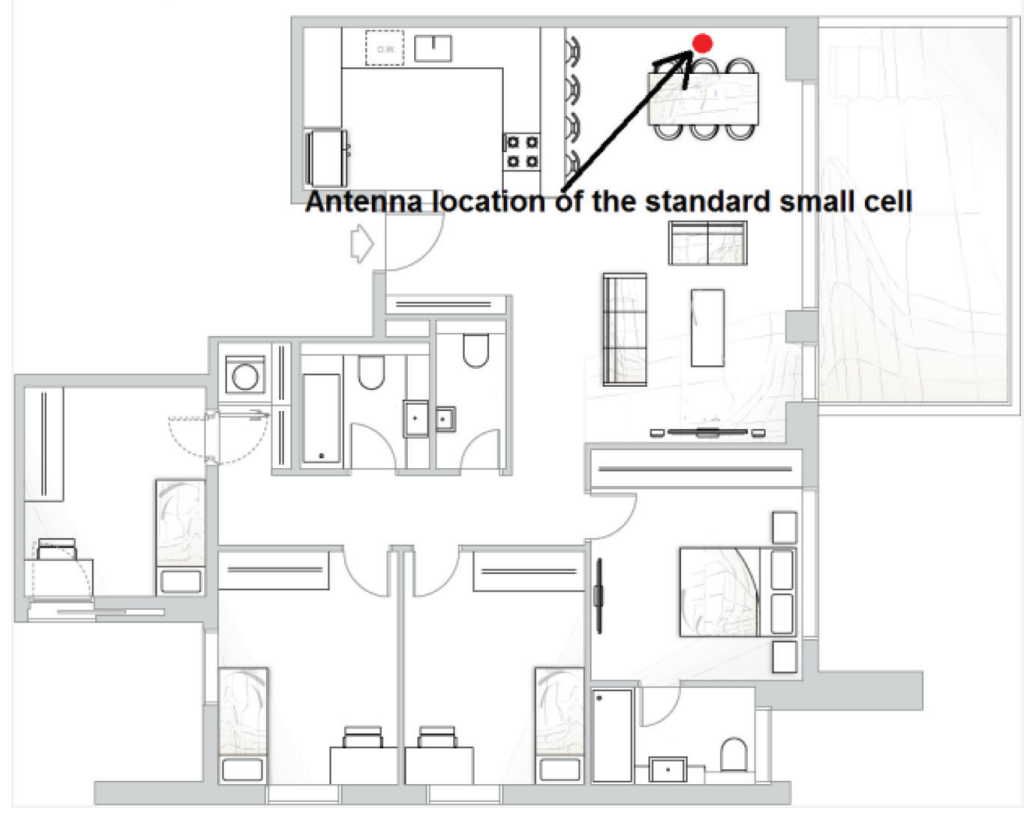

Figure 3 Traditional small-cell installed location.

Figure 4 illustrates the expected capacity of a traditional small-cell in the indoor scenario. The capacity map is obtained by simulating the expected end-user capacity at every given location within the indoor environment.

In order to evaluate the Green small-cell architecture, it is necessary to decide the number of RRHs, their placement and respective transmit power. This process involves a tradeoff between inherently contradictory design considerations. Therefore, the goal was to achieve a suboptimal deployment, based on minimizing the transmit power and number of RRHs, while achieving similar performance in terms of capacity and coverage as the traditional small cell reference scenario.

Figure 5 illustrates the impact of the number of RRHs on the overall coverage.

The location of the RRHs is illustrated in the lower right corner of Figure 5. In the upper left corner of the figure, the overall capacity when using only RRH1 is demonstrated. It can be seen that there is very good coverage only in the vicinity of RRH1, which is the upper right side of the apartment, while the lower left side has no service. In the upper right corner of Figure 5, the overall capacity when using both RRH1 and RRH2 is demonstrated. 


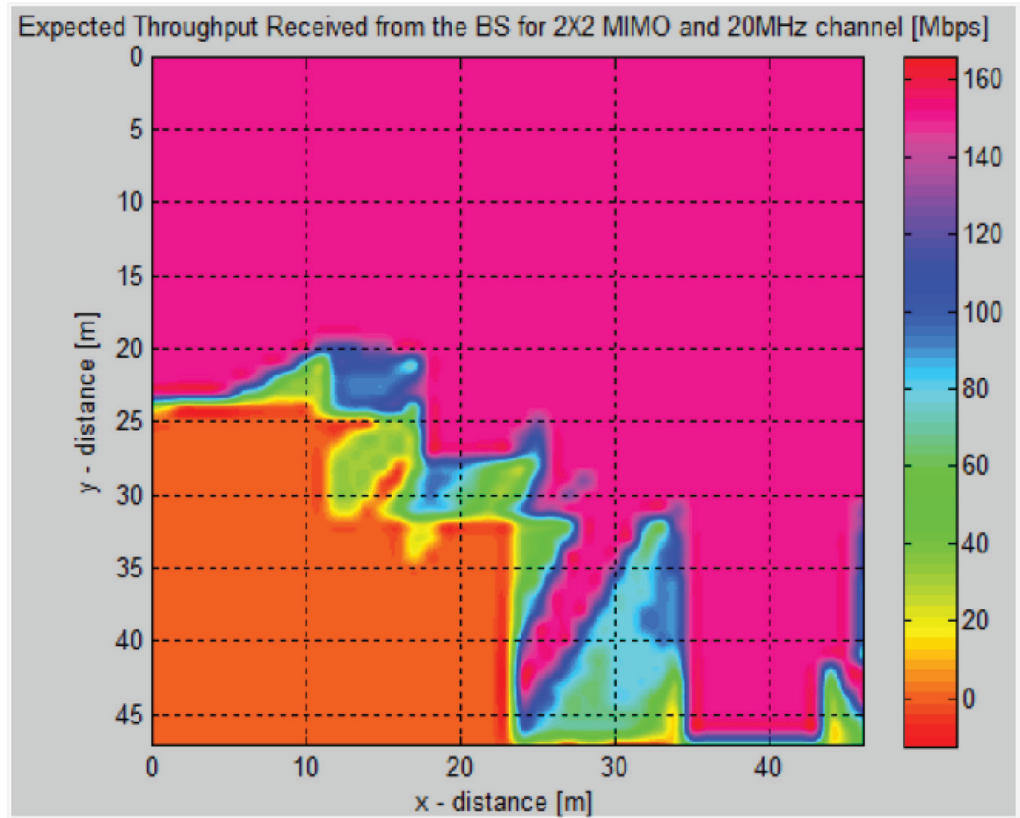

Figure 4 Traditional small-cell capacity results.

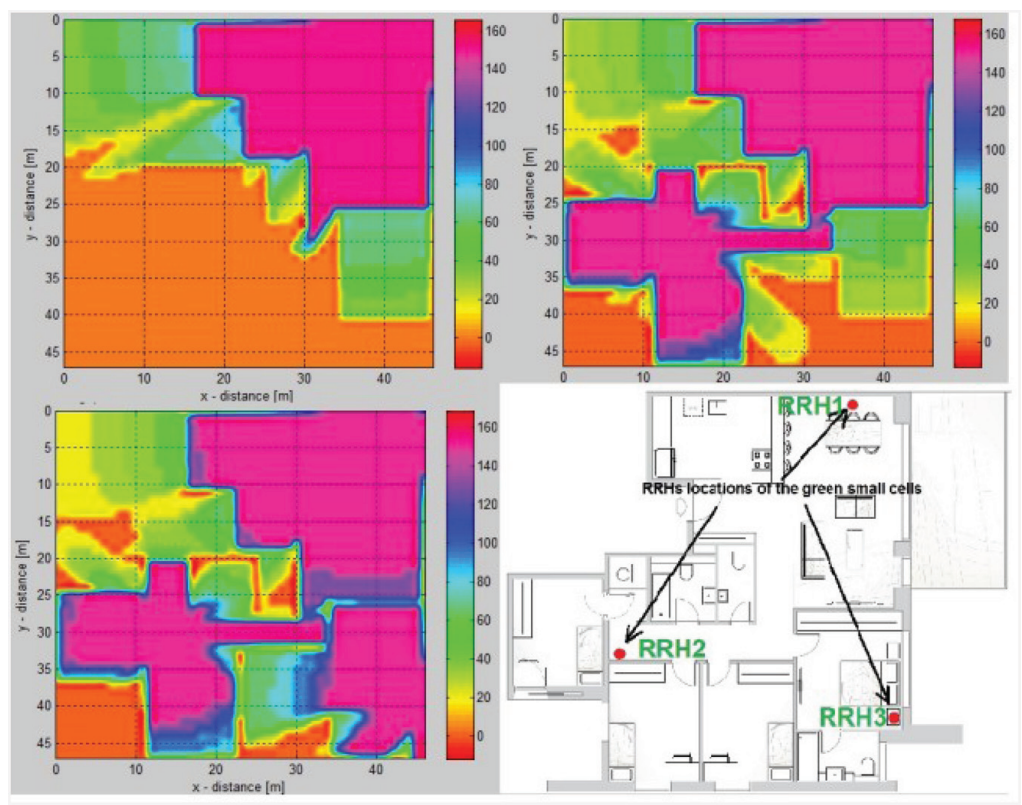

Figure 5 Impact of number of RRHs. 
RRH2 was installed in the main area, where RRH1 could not deliver service. The coverage improvement is clearly evident, even though the lower right side of the apartment experiences significant capacity degradation compared to most of the apartment. Finally, in the lower left corner of Figure 5, the overall capacity when using RRH1 to RRH3 is demonstrated. RRH3 was installed in an area where both RRH1 and RRH2 could not deliver full capacity. Again, the coverage improvement is clearly evident in the vicinity of RRH3. In all of the installation scenarios, the transmit power of each RRH is $-20 \mathrm{dBm}$, which is $40 \mathrm{~dB}$ less than the transmit power of the traditional small-cell.

Figure 6 illustrates the impact of RRH transmit power on the overall coverage.

The impact of RRH transmit power on the RRH location is illustrated in Figure 6. In the upper left corner of Figure 6, the overall capacity when using 4 RRHs with a transmit power of $-15 \mathrm{dBm}$ is demonstrated. It can be seen that theoretically there is very good coverage across all of the apartment, but at the expense of potential interference between the RRHs. In the upper right corner of the Figure, the overall capacity when using 4 RRHs with a transmitted power of $-27 \mathrm{dBm}$ is demonstrated. In this case, the potential mutual interferences

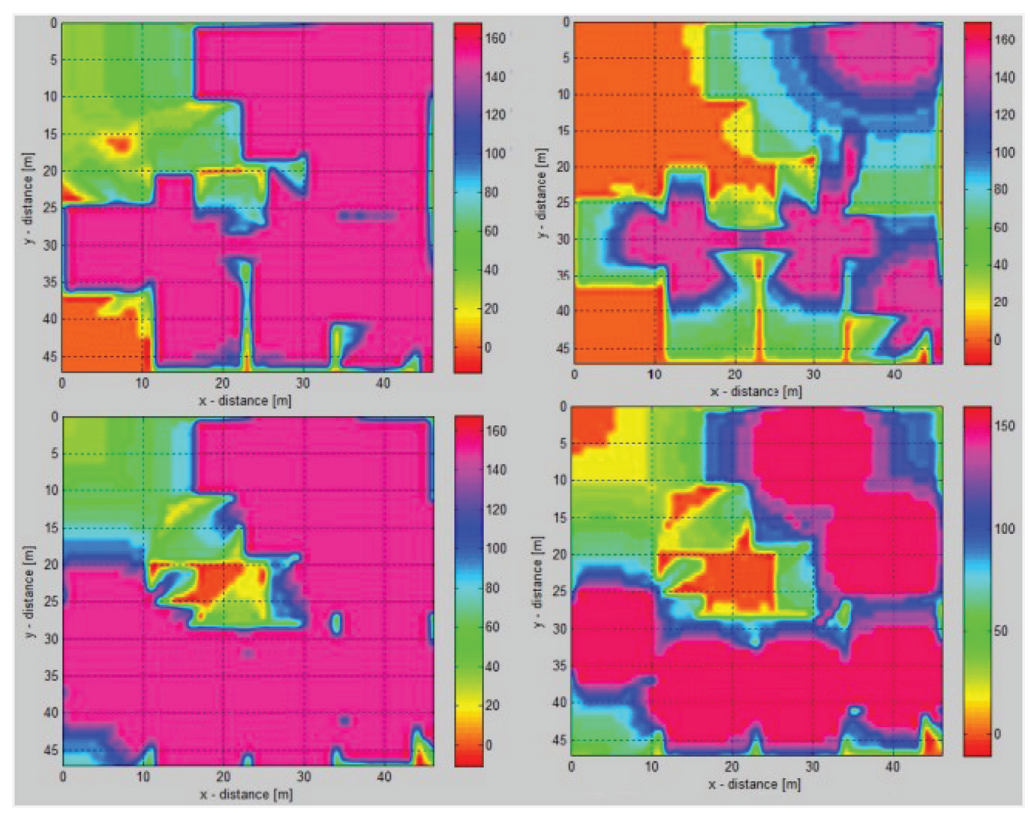

Figure 6 Impact of reduction in RRHs transmitted power on installtion. 
between the RRHs is reduced, but at the expense of the coverage performance. In the lower left corner of the Figure, the overall capacity when using 6 RRHs with a transmitted power of $-20 \mathrm{dBm}$ is demonstrated. It can be seen that theoretically there is a very good coverage across the entire apartment, but at the expense of potential interference between the RRHs.

When this scenario is compared with that of 4 RRHs with transmit power of $-15 \mathrm{dBm}$, it is observed that the capacity and potential mutual interference between the RRHs are comparable with the case with two additional RRHs. This contributes to the reduction in the RRH transmitted power by an additional $5 \mathrm{~dB}$. Finally, in the lower right corner of Figure 6 the overall capacity when using 6 RRHs with a transmitted power of $-27 \mathrm{dBm}$ is demonstrated. This time the potential mutual interferences between the RRHs is reduced and the overall coverage is reasonable but on the expense of addition of 2 extra RRHs. In conclusion, Figures 5 and 6 demonstrate some of the basic installation considerations and potential advantage of the green small-cell architecture.

Figure 7 demonstrates the location of the RRHs in the green small-cell installation in an indoor scenario. In the suggested configuration each RRH transmitted power was $-20 \mathrm{dBm}$, which is $40 \mathrm{~dB}$ below the transmitted power of the traditional small-cell.

Figure 8 demonstrates the expected capacity of a green small-cell in the indoor scenario.

The simulation results clearly show that even when the power levels from the radio head of the green small-cell were decreased by $40 \mathrm{~dB}$ compared to the traditional small-cell deployment the capacity of the suggested architecture was at least as high as the capacity achieved in the traditional scenario, while in extreme scenarios the traditional small-cell could not guarantee any service whereas the green small-cell delivered the maximal capacity. This was practically achieved for all the relevant locations within the indoor.

The green small cell can further leverage the CPRI protocol to achieve even better performance compared to the traditional small cell by utilizing the CPRI embedded control functionalities concepts. The CPRI protocol supports different base station configurations in terms of "antenna-carrier". "antennacarrier" includes all the data of one carrier at one independent antenna element which is typically characterized by having exactly one antenna connector to the RRH. With the concept of controlling each carrier at each antenna element, a more advanced RRH management can be implemented. In the reference scenario depicted in Figure 7, four RRH were installed in the given indoor scenario. Each RRH used 5 channels (carriers) with a $2 \times 2$ MIMO 


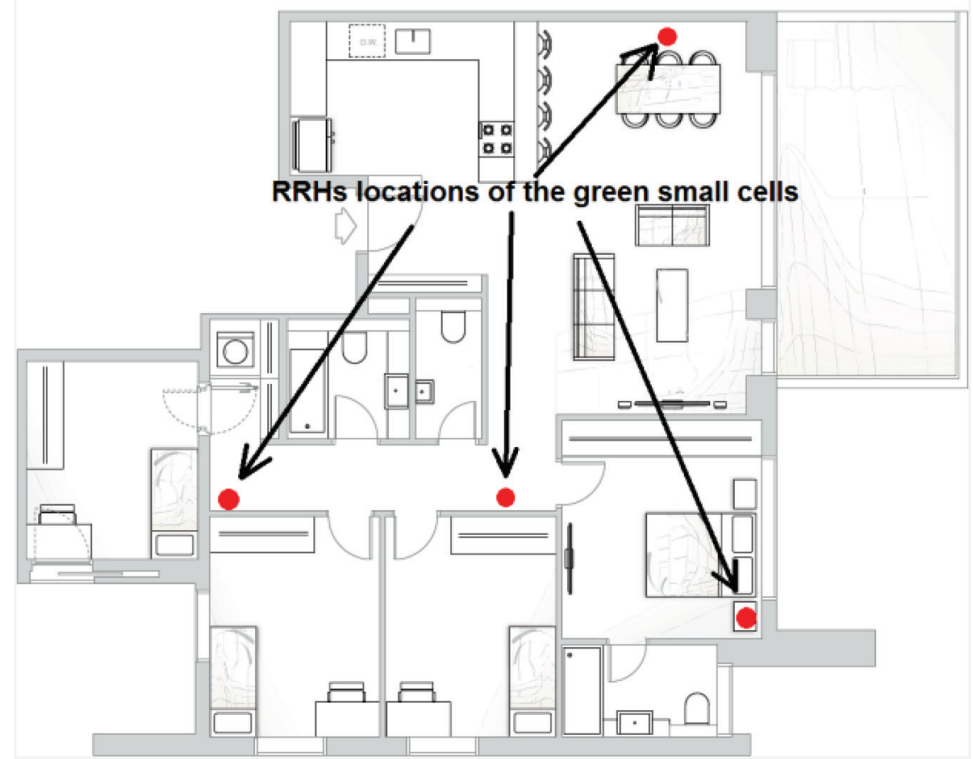

Figure 7 Green small-cell installed locations.

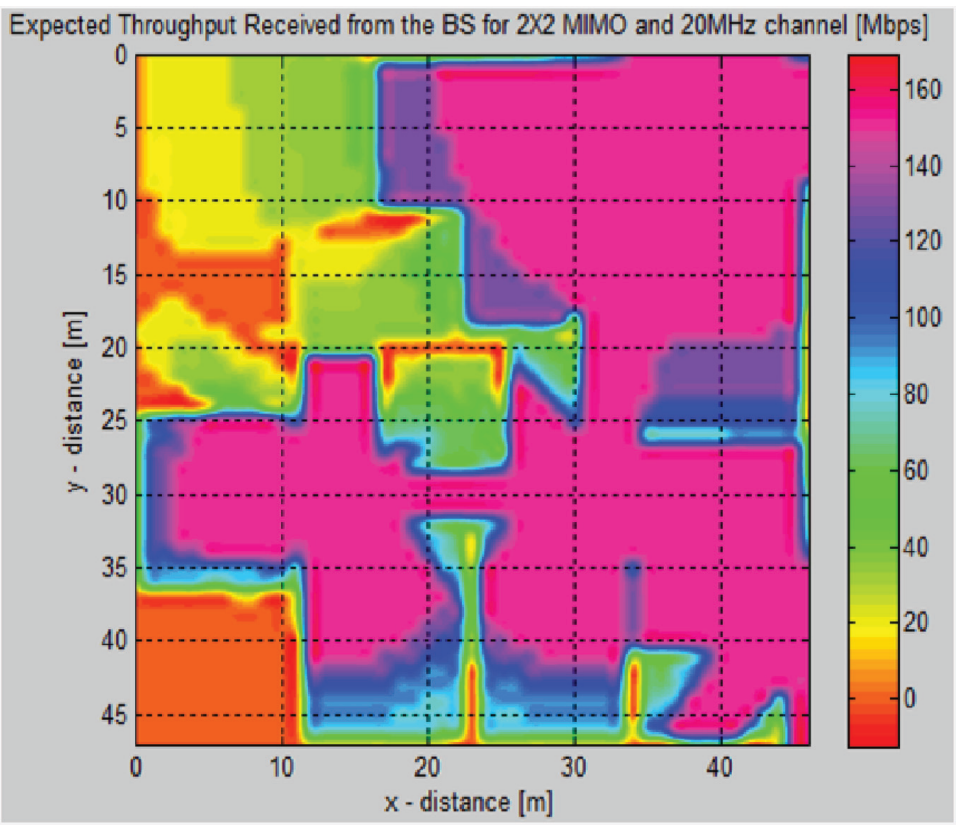

Figure 8 Green small-cell capacity results. 
configuration summing to a total of 10 independent transmitters and 10 independent receivers in order to achieve the maximum capacity potential in every possible locations of the end user within the indoor environment. In practice achieving this goal is not necessary at all times. In the case of a typical office environment this goal will be normally required during most of the working hours. However while the office is closed the base stations will be extremely underutilized and easing the requirements from the base stations to enable only the minimum capacity potential in every possible locations of the end user within the indoor environment is more optimal. Thus, enabling the BBU to control the number of carriers used at each antenna element in any given time can further increase the energy efficiency of the green small cell architecture. In the U.S a total of 251 working days is expected for 2016, during these days each RRH can be configured to operate at minimum configuration, i.e., one carrier with only one transmitter. This configuration reduces the reference scenario configuration from a total of 10 independent transmitters and 10 independent receivers to only one transmitter and one receiver for $31.28 \%$ of the time. During the working days it is reasonable to assume that the typical office will be closed for at least $33 \%$ of the time in any given day, this effectively means that $51.86 \%$ of the time the base station could operate at minimum configuration which is only at $10 \%$ of its energy consumption at peak hours, effectively equivalent to reducing the transmitted average power levels by additional $13 \mathrm{~dB}$ which amounts to a total of $53 \mathrm{~dB}$ power difference between the green small cell and the traditional small cell. A similar logic can be applied to residential indoor scenarios. This calculation demonstrate how a basic automatic algorithm based on bandwidth demand can further optimize the energy consumption of the green small cell compared to the traditional small cell and increase the benefits of the green small cell architecture.

\section{Future Work and Work In Progress}

The simulation results and analysis demonstrated a dramatic improvement of $40 \mathrm{~dB}$ in the electromagnetic emission levels with the suggested architecture. Although the power reduction is significant there is still room for additional improvement. The next step in the evolution of the suggested architecture calls for the usage of additional technologies, among the most relevant are: Next Generation Fronthaul Interface, Visible Light Communication and 802.11. 


\subsection{Next Generation Fronthaul Interface (NGFI)}

The current available protocol for the fronthaul interface is based on the CPRI (V7.0). The current CPRI drawback is its inefficiency which is demonstrated in its sample width and with the constant availability of the link. The sample width normally used for LTE is 15 bits for each one of the In-phase and Quadrature signals while in practice the LTE requires only 7 bits for the Inphase and 7 bits for the Quadrature signals. Therefore, with proper design the requirement from the fronthaul for a fully loaded LTE-A cell can be reduced to about $50 \mathrm{Gbps}$. Additional techniques can be used to increase the efficiency of the fronthaul by using lossless compression techniques that exploits the unused subcarriers in the frequency domain, as well as the redundant precoding information in the DL to achieve compression ratios of 3:1 even in full load conditions [19]. The constant availability property of the CPRI protocol results in that the CPRI links are always ON and therefore consume energy even when there is no data to deliver over the fronthaul. A packet switch concept for the fronthaul interface will increase the energy efficiency on the frontahul link relatively to the actual data usage over the link. Currently CPRI cooperation is working on increasing the protocol efficiency and the inclusion of new features.

Simultaneously, standardization activities have been started within the IEEE with the adaptation of the Time-Sensitive-Network (TSN) IEEE 802.1CM standard [20], the IEEE P1904.3 Radio over Ethernet (RoE) standard [21] and latest Next Generation Fronthaul Interface (NGFI) IEEE P1914.1 initiatives [22]. The IEEE P1904.3 is aimed at taking advantage of the Ethernet developments and specifies a scalable and streamlined packet based radio interface solution that complements the existing CPRI radio transport specification based on fixed time division-multiplexing. The IEEE P1914.1 Working Group standardization effort proposal lies on the function re-split between BBU and RRH and the new Next Generation Fronthaul Interface (NGFI). The purpose of the IEEE P1914.1 is to support the Fronthaul long term goal of enabling a traffic adaptive interface with support for statistical multiplexing and small cell coordination while being antenna and radio interface neutral. IEEE P1914.1 will study the possible implementation options by leveraging the CPRI encapsulation over Ethernet or revisiting the Layer-1 partitioning between baseband and RRHs modules traffic adaptive bit rates. Enabling CPRI over Ethernet will require an additional function which can encapsulate the CPRI frame in an appropriate manner, this encapsulation is under IEEE P1904.3 development. Nevertheless, the packetized networks are non-deterministic and lack precise time synchronization, which is inherent 
and required for CPRI. Highly accurate clock distribution and Time Sensitive Networking (TSN) services have to be implemented, in order to fulfill the strict requirements of the Future mobile Fronthaul network architecture. The IEEE 802.1CM Time-Sensitive Networking for Fronthaul is in progress to allow time-synchronized low latency high bandwidth services over Ethernet networks.

\subsection{Visible Light Communication}

The Visible Light Communication (VLC) is a technology that exploit light rather than the traditional Radio Frequency (RF) waves to transfer information. Since the VLC uses light as means of communications than the VLC is the perfect Green Radio in the sense that its exposure to electromagnetic radiation is zero.

The next step in the evolution of the suggested architecture in this paper is to incorporate the VLC and packetized based fronthaul interface such as the IEEE 1904.3 based protocol. Since the suggested architecture is agnostic to the fronthaul interface the conceptually incorporation of the new fronthaul protocol in the suggested architecture is expected to be a straight forward but only when such interface will be defined it will be possible to analyze its impact on the suggested architecture. The VLC building blocks on the other hand are generally known and available today.

The VLC was standardized under the IEEE 802.15.7 standard back in 2011 and achieved maximum rate of 96 Mbps. In 2015 a new activity to update the standard was started under the IEEE 802.15.7r1 [23] working group, this working group defined several use cases for VLC including the Indoor Office/Home Applications. Currently, the working group is aiming towards a next-generation optical wireless standard data rate targets of up to $10 \mathrm{Gbps}$ $[24,25]$. The IEEE $802.15 .7 \mathrm{r} 1$ working group is expecting to release the IEEE $802.15 .7 \mathrm{r} 1$ standard for publication by 2018. The VLC technology is constantly evolving and recent publications suggest that VLC could perform well in indoor scenarios with multi-gigabits rates even achieving rates of 20 Gbps under mobility conditions [26].

The concept of the evolved architecture is to use the VLC for the DL, thus potentially eliminating the electromagnetic radiation from the cell. The current drawback of the VLC technology is that it is not yet practical to use it for the $\mathrm{UL}$ for the $5 \mathrm{G}$ indoor use case. The suggested solution for this drawback is to combine the VLC with the LTE technology while the VLC target is to transmit data in the order of 5 Gbps. The combined solution is illustrated in Figure 9. 


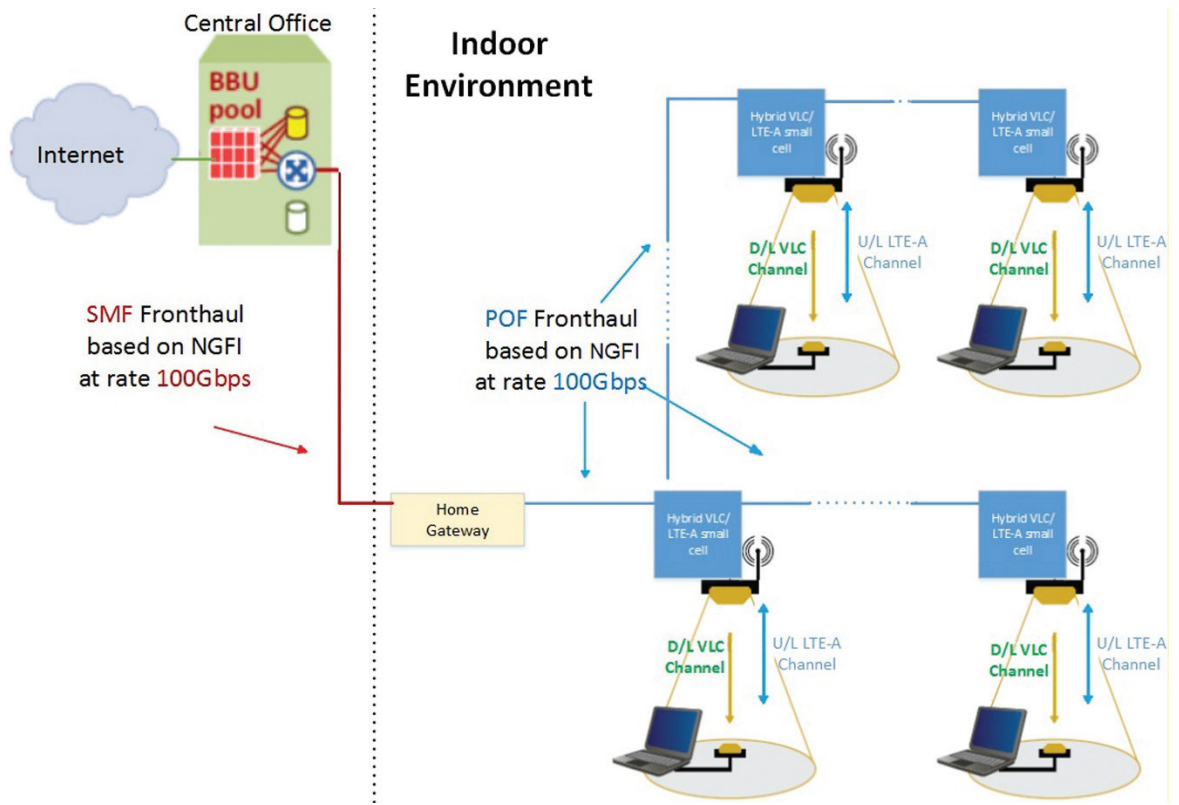

Figure 9 Suggested evolved architecture.

As can be seen in Figure 9 the VLC is used for the DL and the LTE RRH is used for the UL. Since the LTE RRH is used for the UL than the only requirement from the LTE DL is to deliver the UL control information of the UL channel to the User Equipment (UE), this means that it should be possible to properly decode the UL control information at the UE across all over the desired coverage area. Since the control information of the UL channel is basic and critical information, it is transmitted from the base-station in lower modulation and code scheme. This means that for proper decoding of this information the desired Signal to Noise Ratio (SNR) at the UE is relatively low, in the order of $0 \mathrm{~dB}$, meaning that in order to maintain the UL it is enough to achieve this low SNR in the DL across all over the desired area without impacting the overall wireless performance of the cell. Therefore, when using the VLC for the DL the target is to achieve the minimum required SNR to maintain the UL channel across all over the cell coverage area. Figure 10 demonstrates the expected capacity of a green small-cell in the indoor scenario when further reducing the power by $10 \mathrm{~dB}$ compared to the green small-cell concept described in Section 3. 


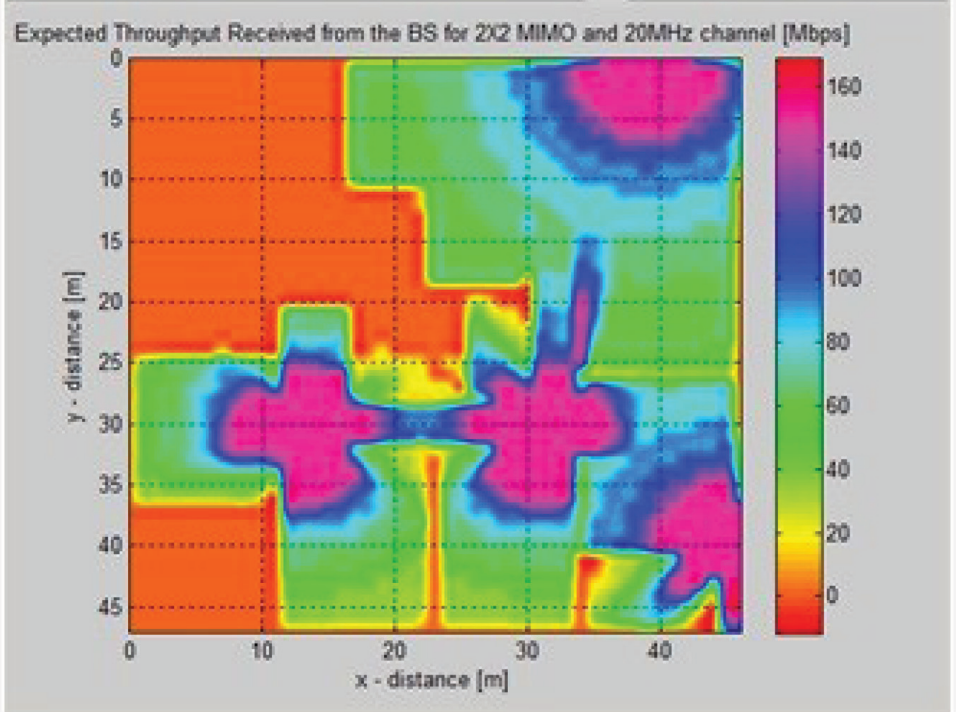

Figure 10 Green small-cell capacity results for maintaining the LTE UL channel concept.

The simulation results clearly shows that even when the power levels from the radio head of the green small-cell were decreased by $50 \mathrm{~dB}$ compared to the traditional small-cell deployment, the DL channel coverage is more than enough to maintain the UL. This was practically achieved for all the relevant locations within the indoor. The concept of operation that is using the VLC as the only DL channel for data is more suitable when the objective is to reduce the exposure to electromagnetic radiation on the expense of data rates delivered. When the data rates delivery for the UE are not met than the configuration can be changed on demand to a different data rate driven concept of operation. In the data rate driven concept of operation the VLC and the LTE are used simultaneously. In this concept the suggested architecture as described in Section 3 is used but at the same time the VLC is used to boost up the overall data rate in the DL by combining the data rates of the LTE cell and the VLC cell.

\subsection{IEEE 802.11 Enhancements}

The IEEE 802.11 standard is an established standard that is constantly evolving based on the evolved market requirements. For our purposes there are two main potential candidates of the IEEE 802.11 family which can potentially 
used to enhance the suggested architecture performance in terms of capacity, availability and reliability. The first candidate is the 802.11ad which is a technology for the $60 \mathrm{GHz}$ band with maximum expected physical layer rate of about $6.8 \mathrm{Gbps}$ with a bandwidth of $2.16 \mathrm{GHz}$ [27]. The second candidate is the 802.11ax which is under development standard for the next generation of the 802.11 at the $5 \mathrm{GHz}$ band, this standard is expected to be released at 2019 with specification of maximum expected physical layer rate of about $2.4 \mathrm{Gbps}$ with a bandwidth of $160 \mathrm{MHz}$ and two streams [28]. The advantage of the 802.11ax over the 802.11ad standard is that it is using the more explored $5 \mathrm{GHz}$ band in a relatively high efficiency while the advantage of the $802.11 \mathrm{ad}$ over the $802.11 \mathrm{ax}$ standard is that typically the $60 \mathrm{GHz}$ signal cannot penetrate walls but can propagate off reflections from walls, ceilings, floors and objects, therefore this property not only exploit the architecture concept of isolating small areas of service in order to increase the system capacity and at the same time reduce the transmitted power, but it also increase the security level of the network.

Figure 11 illustrates the full configuration of the suggested evolved architecture which includes the LTE, VLC and 802.11 technologies:

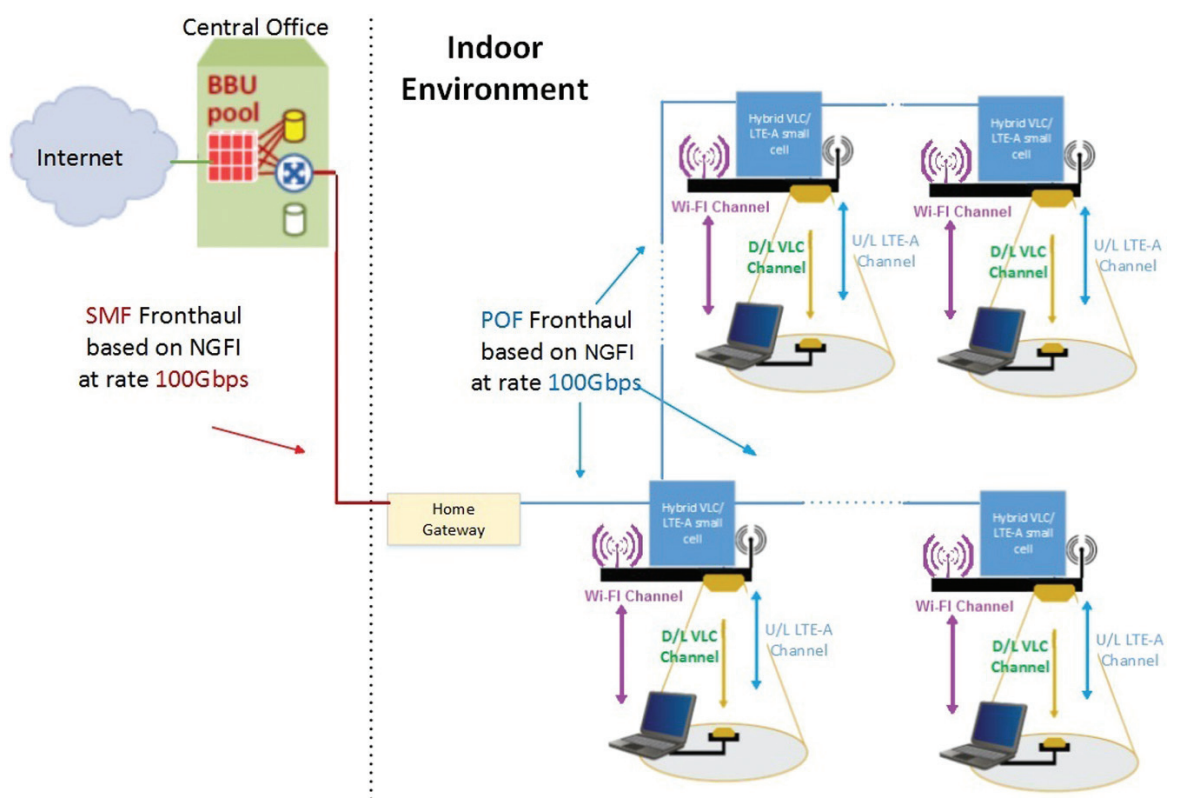

Figure 11 Suggested full evolved architecture. 
Both technologies can support operation at lower frequency bands. While the 802.11ax support the $2.4 \mathrm{GHz}$ band mainly for legacy interoperability reasons, the $802.11 \mathrm{ad}$ supports the $5 \mathrm{GHz}$ and $2.4 \mathrm{GHz}$ mainly for roaming between rooms, the protocol can switch to make use of the lower bands at a much lower rate, in order to achieve smooth handover between RRHs by taking advantage of the lower bands property of propagation through walls. As for the case of LTE technology the 802.11 can be a complementary solution for the VLC in the UL or as a backup or a booster for the DL. The suggested full architecture configuration is highly robust and allow data rate compliant to the $5 \mathrm{G}$ while reducing the electromagnetic radiation significantly below the traditional small cell configuration for indoor scenarios.

\section{Conclusion}

The simulation results and analysis demonstrated a dramatic improvement of $\sim 40 \mathrm{~dB}$ in the electromagnetic emission levels with the suggested architecture. Further improvement in terms of power reduction was suggested with the introduction of VLC as the main means of transporting the Downlink traffic. The next step in the evolution of the suggested architecture calls for the usage of additional technologies, among the most relevant are: Next Generation Fronthaul Interface and 802.11. The suggested evolved architecture combines several radio access technologies in the RRH, thus allowing significant improvement in power reduction and data rates. Moreover, the architecture concept of isolating small areas of service in order to increase the system capacity and at the same time reduce the transmitted power results in increase in the security level of the network. The multi-RAT concept also contributes to the flexibility and reliability of the air-interface. Another aspect of the Evolved architecture includes the NGFI over the fiber links. The usage of the NGFI allows high utilization of the fiber links on top of the high flexibility in the Radio Access Network. The high utilization of the NGFI contributes not only to the potential reduction in the CAPEX but also in energy savings across the network. The combination of multi-RAT for the air-interface and NGFI over fiber enables the concepts of Software Defined Networking (SDN) and Network Function Virtualization (NFV). These concepts include function splits in the Radio Access Network (RAN) and across the core network. Therefore, the suggested evolved architecture also meets the 5G indoor requirements. 


\section{References}

[1] NGMN Alliance (2015). NGMN 5G White Paper V1.0. Available at: https://www.ngmn.org/uploads/media/NGMN_5G_White_Paper_ V1_0.pdf

[2] Chen, Y., Zhang, S., Xu, S., and Ye Li, G. (2011). Fundamental trade-offs on green wireless networks. IEEE Commun. Mag. 49, 30-37.

[3] http://www.greentouch.org

[4] 5G -PPP Architecture Working Group (2016). View on 5G Architecture Version 1.0. Available at: https://5g-ppp.eu/wp-content/uploads/2014/02/ 5G-PPP-5G-Architecture-WP-July-2016.pdf

[5] Next Genetation Fronthaul Interface White Paper V1.0. Available at: http://labs.chinamobile.com/cran/wp-content/uploads/2015/09/NGFIWhitepaper_EN_v1.0_201509291.pdf

[6] China Mobile Research Institute (2011). "C-RAN The Road towards Green RAN Version 2.5". Hong Kong: China Mobile Research Institute.

[7] Chih-Lin, I., Rowell, C., Shuangfeng, H., Zhikun, X., Gang, L., and Zhengang, P. (2014) Toward green and soft: a 5G perspective. IEEE Commun. Mag. 52, 66-73.

[8] Ghodsi, A., Koponen, T., Raghavan, B., Shenker, S., Singla, A., and Wilcox, J. (2011). "Information-centric networking: seeing the forest for the trees," in Proceedings of the ACM Workshop on Hot Topics in Networks (HotNets-X), Cambridge, MA.

[9] Hejie, Y. (2011). Optical Techniques for Broadband In-Building Networks, Ph.d. Thesis, Eindhoven University of Technology, Eindhoven.

[10] Shi, Y. (2013). Optical Broadband In-Home Networks for Converged Service Delivery. Ph.d. Thesis, Eindhoven University of Technology, Eindhoven.

[11] Gwen-Hyung, G., Lauren, K., and Kahn, J. M. (2011). Rate-adaptive coding for optical fiber transmission Systems. J. Lightwave Technol. 29

[12] Smith, B. P. (2011). Error-Correcting Codes for Fiber-Optic Communication Systems. Ph.d. Thesis, University of Toronto, Toronto, ON.

[13] Natasa, Z., and Nenand, R. (2008). "The implementation of coding in optical telecommunication systems," in Proceedings of the 6th WSEAS International Conference on Applied Electromagnetica, Wireless and Optical Communications (ELECTROSCIENCE '08), Trondheim.

[14] Djorddjevic, I. B., Arabaci, M., and Minkov, L. L. (2009). Next generation FEC for high-capacity communication in optical transport network. J. Lightwave Technol. 27, 3518-3530. 
[15] Common Public Radio Interface (CPRI) (2015). CPRI Specification V7.0 (2015-10-09) Interface Specification. Ericsson AB, Huawei Technologies Co. Ltd, NEC Corporation, Alcatel Lucent, and Nokia Networks.

[16] Hamilton, S. A., Robinson, B. S., Murphy, T. E., Savage, S. J., and Ippen, E. P. (2002). $100 \mathrm{~Gb} / \mathrm{s}$ optical time-division multiplexed networks. J. Lightwave Technol. 20:2086.

[17] Shao, Y., Cao, R., Huang, Y., Ji P., and Zhang, S. (2012). “112-Gb/s transmission over $100 \mathrm{~m}$ graded-index plastic optical fiber for optical data center applications," in Proceedings of the Optical Fiber Communication Conference and Exposition and the National Fiber-optic Engineers Conference (OFN/NFOEC), Los Angeles, CA.

[18] Kyösti, P., Meinilä, J., Hentilä, L., Zhao, X., Jämsä, T., Schneider, C., et al. (2007) IST-4-027756 WINNER II D1.1.2 V1.1 WINNER II Channel Models. Information Society Technology.

[19] Lorca, J., and Cucala, L. (2013). "Lossless Compression Technique for the Fronthaul of LTE/LTE-Advanced Cloud-RAN Architecture" in Proceedings of the IEEE 14th International Symposium and Workshops on World of Wireless, Mobile and Multimedia Networks (WoWMoM), Piscataway, NJ.

[20] IEEE 802.1CM. Time - Sensitive Networking for Fronthaul. (2015). Available at: http://www.ieee802.org/1/pages $/ 802.1 \mathrm{~cm} . \mathrm{html}$

[21] IEEE 1904. Access Networks Working Group. Available at: http:// www.ieee 1904.org/index.shtml

[22] IEEE P1914.1. Working Group for Next Generation Fronthaul Interface. Available at: https://standards.ieee.org/develop/project/1914.1.html

[23] Jang, Y. M. (2015) "Current Status of IEEE 802.15.7r1 OWC Standardization," in Proceedings of the International Conference and Exhibition on Visible Light Communications 2015, Yokohama.

[24] TG7r1, Technical Considerations Document, IEEE 802.15 Std., Rev3. Available at: https://mentor.ieee.org/802.15/dcn/15/15-15-049203-0007a-technical-considerations-document.docx

[25] Jungnickel, V., Uysal, M., Serafimovski, N., Baykas, T., O’Brien, D., Ciaramella, E., et al. (2015). "A European view on the next generation optical wireless communication standard," in IEEE Conference on Standards for Communications and Networking (CSCN), Heidelberg.

[26] Hussein, A. T., Alresheedi, M. T., and Elmirghani, M. H. (2015). " 20 Gb/s mobile indoor visible light communication system employing beam steering and computer generated holograms. J. Lightwave Technol. 33, 5242-5260. 
[27] IEEE std 802.11ad-2012

[28] IEEE 802.11-16/0024r1, Proposed TGax draft specification.

[29] NTT DOCOMO (2014). DOCOMO $5 G$ White. Available at: https://www. nttdocomo.co.jp/english/binary/pdf/corporate/technology/whitepaper_ 5g/DOCOMO_5G_White_Paper.pdf

[30] Fang, C., Yu, F. R., Huang, T., Liu, J., and Liu, Y. (2015). A survey of green information-centric networking: Research issues and challenges. IEEE Commun. Surv. Tuts., 17, 1455-1472.

[31] (2011). C-RAN the Road Towards Green RAN. Beijing: China Mobile Research Institute.

[32] Guan, H., Kolding, T., and Merz, P. (2010). Discovery of Cloud-RAN. Zoetermeer: Nokia Siemens Netw.

[33] Lin, Y., Shao, L., Zhu, Z., Wang, Q., and Sabhikhi, R. K. (2010). Wireless network cloud: Architecture and system requirements. IBM J. Res. Develop. 54, 4:1-4:12.

[34] (2011). "ZTE Green Technology Innovations White Paper." Shenzhen: ZTE Corporation.

[35] (2011). Intel Heterogenous Network Solution Brief. Santa Clara, CA: Intel solution brief.

[36] Deliverable D2.1 Business Models and System Requirements for the COMET (COntent Mediator architecture for content-aware nETworks) System, European Seventh Framework Project FP7-2010-ICT -248784STREP.

\section{Biographies}

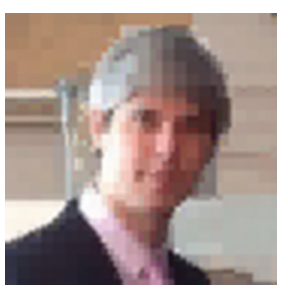

Y. Hazan Holds B.Sc. [05] and M.Sc. [16] from Holon Institute of Technology Israel, all in Electrical Engineering. Yaron has more than 15 years of experience in state of the art communication systems in several communication topics including: broadband access and communication technologies including 802.11 and 802.16 systems. Yaron current research interests include advanced green small cells under fiber-wireless channels and 5G systems. 


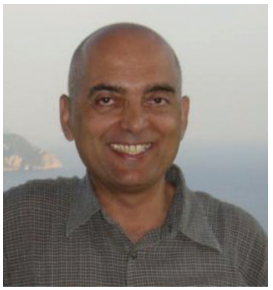

M. Ran (M'86-SM'99) B.Sc. from Ben-Gurion University [78], M.Sc. [83] and Ph.D. [96] from Tel Aviv University Israel, all in Electrical Engineering. Dr. Ran has 25 years experience in state of the art communications systems. Vast experience in development and leadership of $R \& D$ groups in several communication topics including: broadband access technologies, wireless communications including $3 \mathrm{G}$ and $4 \mathrm{G}$ systems, Short-range communications with focus on Ultra-wideband (UWB) technologies, error correction codes and algorithms to improve performances of communications systems. In recent years he has been investigating convergence of optical fibre with wireless technologies, with focus on UWB radio over optical fiber.

Dr. Ran is the CEO of MostlyTek ltd. since 2001 and a lecturer in Holon Institute of Technology (HIT) since 2001. Since 2016 he is also with National Authority of Technological Innovation of Israel.

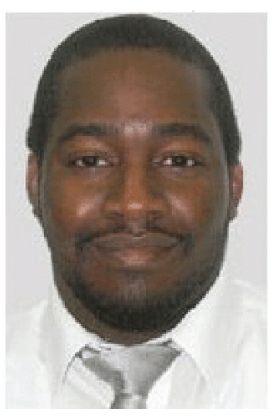

E. Omiyi received his B.Eng. from University of Manchester UK, and $\mathrm{Ph}$.D. from Leeds University UK, all in Electrical and Electronic Engineering. Dr. Omiyi has over a decade of consulting and pioneering research experience in wireless communications, including in $3 \mathrm{G} / 4 \mathrm{G} / 5 \mathrm{G}$ communications. This includes research into self-organizing radio-access networks, device-to-device communications and heterogeneous wireless networks.

Dr. Omiyi is a researcher and lecturer in Holon Institute of Technology (HIT) since 2016. 
